\title{
A Comprehensive Investigation of Color Models used in Image Processing
}

\author{
Kondekar Vipul H. \\ PhD Research Student \\ Department of Electronics and telecommunication \\ Engineering C.O.E., Pandharpur, Maharastra State, \\ India
}

\author{
Bodhe S. K. \\ PhD Research Guide \\ Department of Electronics and telecommunication \\ Engineering C.O.E., Pandharpur, Maharastra State, \\ India
}

\begin{abstract}
This paper is a study of different color models for color image processing. Color has been recognized as an important visual aspect for image and scene analysis. Research work in color image processing has focused on color image formation, color quantization, human visual perception, image segmentation, color-based object recognition, and image database retrieval. RGB color space is generally adopted by image acquisition device, while other kinds of color space are derived from RGB space by using either linear or nonlinear transformations. The choice of a color space is important for many computer image processing algorithms (e.g., feature detection, image classification, object recognition, and visual tracking). No color space can be considered as universal because color can be interpreted and modeled in different ways. With the large variety of available color spaces the inevitable question that arises is how to select the color model that produces the best result for a particular computer vision task. In this paper, the main aim is to survey the theory of different color spaces since the performance of an image analysis procedure is known to depend on the choice of the color space.
\end{abstract}

\section{General Terms}

Colour spaces, Colour Models, Transformations

\section{Keywords}

RGB, HSV, CMYK, YCbCr , CIE; Space transformation.

\section{INTRODUCTION}

Color provides humans with much useful information for object classification, sorting, inspection, verification, and anomaly detection. Over the past few years, color machine vision systems have been introduced for the purpose of automating such operations. Color image processing can be done using different color models, a color model is an abstract mathematical model describing the way colors can be represented as tuples of numbers, typically as three or four values or color components. When this model is associated with a precise description of how the components are to be interpreted (viewing conditions, etc.), the resulting set of colors is called color space.

\section{DIFFERENT COLOR SPACES}

Choice of the best performing color space has been an open Question in recent years as using one space instead of another, can bring considerable improvements in certain applications of image processing. A color model (or color space) is a way of representing colors and their relationship to each other. Different image processing systems use different color models for different reasons. The color picture publishing industry uses the CMY color model. Color CRT monitors and most computer graphics systems use the RGB color model.[1] Systems that must manipulate hue, saturation, and intensity separately use the HSI color model.

A color space specifies colors as tuples of (typically three) numbers, according to certain specifications. Color spaces lend themselves to reproducible representations of color, particularly in digital representations, such as digital printing or digital electronic display. The purpose of a color space is to facilitate the specification of colors in some standard, generally accepted way.

Most classical Color spaces can be grouped into four main families, namely:

The primary color spaces ((R,G,B), (RC,GC,BC), (RE,GE,BE), (RF,GF,BF), (X,Y,Z),etc.).

The Luminance Chrominance color spaces $\left(\left(\mathrm{L}^{*}, \mathrm{a}^{*}, \mathrm{~b}^{*}\right),\left(\mathrm{L}^{*} \mathrm{u}^{*} \mathrm{v}^{*}\right),(\mathrm{Y} 1, \mathrm{U} 1, \mathrm{~V} 1),(\mathrm{Y} 1, \mathrm{I} 1, \mathrm{Q} 1), \mathrm{etc}.\right)$.

The perceptual color spaces ((H,S,I), (L,C,H),(H,L,S),etc.)

The independent axis color spaces((I1,I2,I3)).

\subsection{CLASSIFICATION}

Color spaces are classified into three categories based on the color perception.

2.1.1 Luminance / chrominance-type color space: In this kind space, color information includes three components. One component responds the non-color perception, the other two components respond the color perception. When gray image is needed, this color space is very convenient. $L \alpha \beta$ color space belongs to this kind, $\mathrm{L}$ is luminance channel, $\alpha$ is yellow-blue channel, $\beta$ is red-green channel.

\subsubsection{Mixture-type color space}

In this kind space, three primary colors are used to synthesize color in proportion. RGB, LMS, and CMY $(\mathrm{K})$ belong to this category.

\subsubsection{Hue / saturation / luminance-type color space}

In this kind space, hue and saturation are used to respond the color perception. Luminance is used to respond the luminance information, e.g. HSL, HSV, and LCH belong to this kind. L $\alpha \beta$ color space has two advantages that it does not dependent on the equipment and has wide color gamut. Because its three channels are approximately orthogonal, it can at its best reduce the influence which comes from the other channels change and avoid the channel overlapping.[2] 


\subsection{4 colour spaces also can have categorization} as

- HVS based colour spaces include the RGB colour space ,the opponent colors theory based colour spaces and the phenomenal colour spaces. These colour spaces are motivated by the properties of theHVS.

- Application specific colour spaces include the colour spaces adopted from TV systems (YUV,YIQ), photo systems (Kodak Photo YCC) and printing systems (CMY (K))

- CIE colour spaces are spaces proposed by the CIE and have some properties of high importance like device-independency and perceptual linearity (CIEXYZ, Lab and Luv)

\section{DIFFERENT COLOR SPACES}

\subsection{The RGB color space}

The RGB color space is the most used color space for computer graphics. Note that R, G, and B stand here for intensities of the Red, Green, and Blue guns in a CRT. It is an additive color space: red, green, and blue light are combined to create other colors. It is not perceptually uniform. The RGB color space can be visualized as a cube. Each color-axis (R, G, and B) is equally important. Therefore, each axis should be quantized with the same precision. So, when the RGB color space is quantized, the number of bins should always be a cube of an integer.

The conversion from a RGB image to a gray value image simply takes the sum of the $\mathrm{R}, \mathrm{G}$, and $\mathrm{B}$ values and divides the result by three.

The RGB color model is standard design of computer graphics systems and is not ideal for all of its applications. The red, green and blue color components are highly correlated. This makes it difficult to execute the image processing algorithms.

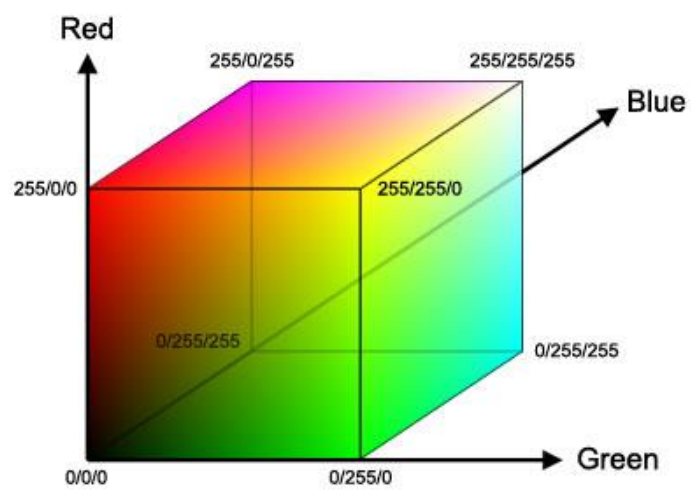

Fig.1 : RGB color model

\subsection{The HSx color spaces}

The HSI, HSV, HSB, and HLS color spaces (conventionally called ' $\mathrm{HSx}$ ') are more closely related to human color perception than the RGB color space, but are still not perceptually uniform.[9]

The axes from the HSx color spaces represent hue, saturation, and lightness (also called value, brightness and intensity) color characteristics. The difference between the different HSx color spaces is their transformation from the RGB color space. They are usually represented by different shapes (e.g., cone, cylinder). HSV color space is visualized as a cone. Hue is the color component of the HSx color spaces. Hue is an angle between a reference line and the color point in RGB space, the range of this value is between 0 and 360, for example blue is 240 .Hue is the attribute of a visual sensation according to which an area appears to be similar to one of the perceived colors, red, yellow, green, and blue, or a combination of two of them. In other words, hue is the color type, such as red or green.

Saturation is the colorfulness of an area judged in proportion to its brightness. In the cone, the saturation is the distance from the center of a circular cross-section of the cone, the 'height' where this cross-section is taken is determined by the Value, which is the distance from the pointed end of the cone. The value is the brightness or luminance of a color, the attribute of a visual sensation according to which an area appears to emit more or less light. When Saturation is set to 0 , Hue is undefined. The Value-axis represents the gray-scale image.

The HSV color space can easily be quantized, the hue is the most significant characteristic of color so this component gets the finest quantization. In the hue circle, the primary colors red, green, and blue, are separated by $120^{\circ}$. The secondary colors, yellow, magenta, and cyan, are also separated by $120^{\circ}$ and are $60^{\circ}$ away from the two nearest primary colors.

The biggest strength of the HSV model, is that it is intuitive, it is easy to describe a color using this model, it makes histogram equalization over all components easier to implement (when equalizing RGB histograms, a thing called a color shift can occur, which means that for instance the green component is distributed differently than the blue component, thus the combined green and blue vector will represent a different color value than the original value of value of the colour).

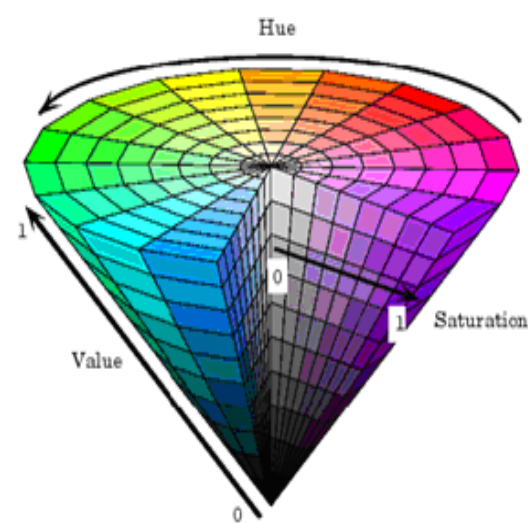

Fig.2 : HSV Color Space

The advantage of HSI is that it decouples color tone from brightness. The hue coordinate is particularly useful for applications that need to distinguish objects with different colors, whereas variations due to irregular object geometry are localized primarily in the intensity coordinate. The HSI color space has been used to implement a color edge detector . 


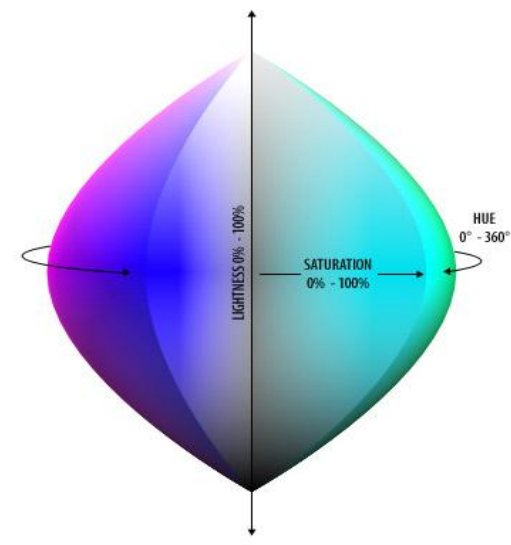

Fig.3 :HSL Color Space

\subsection{The YUV and YIQ color spaces}

The human eye is more sensitive to brightness than to color. Accordingly, color definition is defined by a luminance or brightness (Y) component, an in-phase component (I) and a quadrature component $(\mathrm{Q})$ The YUV and YIQ color spaces are developed for television broadcasting. ' $\mathrm{Y}$ ' is luminance information. It gives requires by the monochrome TV. It is called Gray image, usually used to represent black and white image (monochrome).

YUV models human perception of color in a different way than the standard RGB model used in computer graphics hardware. The human eye has fairly little color sensitivity: the accuracy of the brightness information of the luminance channel has far more impact on the image discerned than that of the other two. YUV model is suitable for application like steganography. 'I', 'Q' are chrominance information where I is called In phase component and Q is called Quadrature phase component.

Luminance: A component, the black and white or brightness element, of an image. It is written as $\mathrm{Y}$, so the $\mathrm{Y}$ in YUV, $\mathrm{YIQ},(\mathrm{Y}, \mathrm{R}-\mathrm{Y}, \mathrm{B}-\mathrm{Y}$ ) and $\mathrm{Y}, \mathrm{Cr}, \mathrm{Cb}$ is the luminance information of the signal. In a colour TV system the luminance signal is usually derived from the RGB signals, originating from a camera, by a matrix or summation of approximately: $\mathrm{Y}=0.3 \mathrm{R}+0.6 \mathrm{G}+0.1 \mathrm{~B}$

Chrominance: The colour part of a signal, relating to the hue and saturation but not to the brightness or luminance of the signal. Thus black, grey and white have no chrominance, but any colored signal has both chrominance and luminance. $\mathrm{Cr}$ and $\mathrm{Cb},(\mathrm{R}-\mathrm{Y})$ and $(\mathrm{B}-\mathrm{Y})$ represent the chrominance information of a signal.

YIQ space is the same as the YUV color space, where the I-Q plane is a $33^{\circ}$ rotation of the UV plane. The $\mathrm{Y}$ signal represents the luminance of a pixel and is the only channel used in black and white television. The U and V for YUV and I and Q for YIQ are the chromatic components.

\subsection{CMYK}

Printing systems more commonly use CMYK, which is a subtractive color system using Cyan, Magenta, Yellow, and black (the "K" in CMYK)."The primary colors of pigments are cyan, yellow, and magenta. These are called the subtractive primary colors because as you add pigments to a white page, they subtract (absorb) more light, and the reflected color becomes darker.'

\subsection{CIE L*A*B}

The Commission International L'Eclairage (CIE) develops device independent color spaces ,it contain a wider color gamut than RGB and CMYK. The size and complexity of the CIE color spaces, including $\mathrm{L} * \mathrm{~A} * \mathrm{~B}$

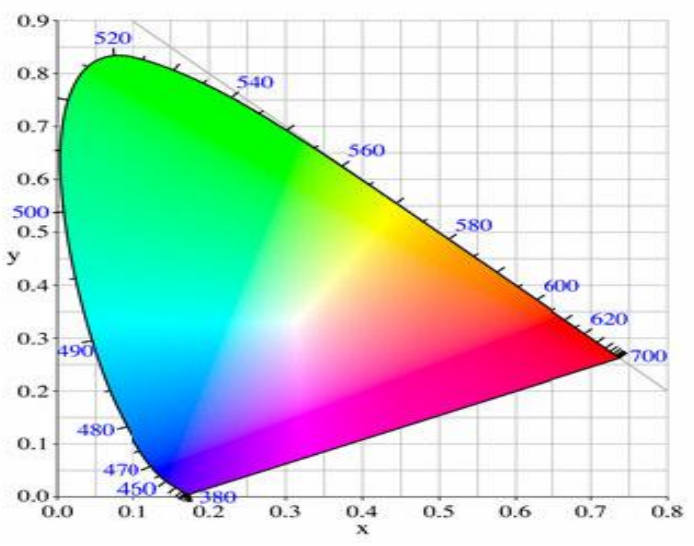

Fig.4 : CIE XYZ color model

\section{6 $\beta \delta \varepsilon$ color space}

This color space establishes a primary axis containing the expected color range of the object. The second and third coordinates contain magnitude and chromaticity of any deviation from the expected color. The separation into application specific coordinates is the main advantage of the new color space The proposed color space takes advantage of highly localized color distributions.

\section{7 $\mathbf{R}_{\mathrm{N}} \mathbf{G}_{\mathrm{N}} \mathbf{B}_{\mathrm{N}}$ color space}

One group of commercial televisions use cathode ray tube with three type of phosphor which create spectrum from each of phosphor is not monochrome and is related to tube manufacturer. Practically, these tubes are compared by standard phosphor introduced by N.T.S.C.

YUV color space is used in SECAM and PAL TV system. YUV is a linear transform of RNGNBN.[3]

\subsection{Double-zero-sum (DZS)}

Definition of the double-zero-sum (DZS)color space as follows:

Definition1. Let $\mathrm{C} 1 \mathrm{C} 2 \mathrm{C} 3$ be a color space defined by a linear Transformation of the RGB color space as following

$[\mathrm{C} 1, \mathrm{C} 2, \mathrm{C} 3]=[\mathrm{R}, \mathrm{G}, \mathrm{B}] \mathrm{C}$

Let the column-sum-vector [b1, b1, b3] be the row vector of the Sum over each column of the transformation matrix $\mathrm{C}$, where bj is the sum of the elements in the $\mathrm{j}$ th column, i.e., $b j=\sum_{i=1}^{3} C i j$.

If Two column-sums are zero while the remaining one is not, the Color space $\mathrm{C} 1 \mathrm{C} 2 \mathrm{C} 3$ is called the double-zerosum (DZS)color space

\subsection{IHLS Color Space}

It is Improved hue luminance and saturation color space. Many color space conversion systems define a saturation coordinate which is dependent on the brightness coordinate of the image and hence are not very suitable for the image analysis applications. To overcome this short coming of conversion systems, an improved saturation measurement is presented in [4]

This improved saturation measurement has following two advantages:

1.It always has small values for a chromatic colors.

2.It is independent of brightness values. 


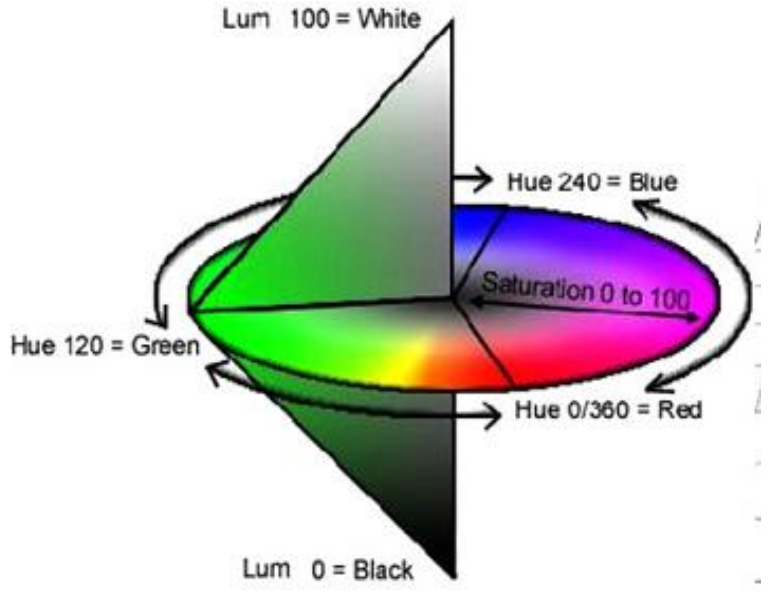

Fig.5: HSL color model

\section{COLOR SPACE CONVERSIONS}

Sometimes it is desirable to be able to convert from one color space to another. In some cases this is an easy process, but in other cases, such as converting from RGB to XYZ, it is a bit more complicated.

\subsubsection{Conversion from RGB to HSV is as following}

$H=\cos ^{-1}\left\{\frac{\frac{1}{2}[(R-G)+(R-B)]}{\sqrt{(R-G)^{2}+(R-B)(G-B)}}\right\}$

$S=1-\frac{3}{R+G+B}[\min (R, G, B)]$

and $V=\frac{1}{3}(R+G+B)$

\subsubsection{Conversion from HSI to RGB is as following}

Case1: $0 \leq H<120$

$$
\begin{aligned}
& R=I\left[1+\frac{S \cos H}{\cos \left(60^{\circ}-H\right)}\right] \\
& G=3 I-(R+B) \\
& B=I(1-S)
\end{aligned}
$$

Case2: $120^{\circ} \leq H<240$

$$
\begin{aligned}
& H=H-120 \\
& R=I(1-S) \\
& G=I\left[1+\frac{S \cos H}{\operatorname{Cos}\left(60^{\circ}-H\right)}\right] \\
& B=3 I-(R+G)
\end{aligned}
$$

Case3: $240^{\circ} \leq H<360$

$H=H-240$

$G=I(1-S)$

$B=I\left[1+\frac{S \cos H}{\operatorname{Cos}\left(60^{\circ}-H\right)}\right]$

$R=3 I-(B+G)$
4.1.3 Conversion from RGB to YIQ is as following

$$
\begin{aligned}
& Y=0.299 R+0.587 G+0.114 B \\
& I=0.596 R-0.275 G-0.321 B \\
& Q=0.212 R-0.523 G+0.311 B
\end{aligned}
$$

4.1.4 Conversion from $R G B$ to $Y I Q$ is as following:

$\mathrm{R}=\mathrm{Y}+0.956 \mathrm{I}+0.621 \mathrm{Q}$

$\mathrm{G}=\mathrm{Y}-0.272 \mathrm{I}-0.647 \mathrm{Q}$

$\mathrm{B}=\mathrm{Y}-1.106 \mathrm{I}+1.703 \mathrm{Q}$

4.1.5 YUV is a linear transform of RNGNBN as follows

$\mathrm{Y}=0.299 \mathrm{R}_{\mathrm{N}}=0.587 \mathrm{G}_{\mathrm{N}}=0.114 \mathrm{~B}_{\mathrm{N}}$

$\mathrm{U}=0.493\left(\mathrm{~B}_{\mathrm{N}}-\mathrm{Y}\right)$

$\mathrm{V}=0.877\left(\mathrm{R}_{\mathrm{N}}-\mathrm{Y}\right)$

$\mathrm{YCbCr}$ color space is very close to YUV space. This space is scaled and shifted YUV color space so as $\mathrm{Cb}$ and $\mathrm{Cr}$ is between range of $(0,1)$ and are obtained as follows,

$\mathrm{C}_{\mathrm{b}}=\left(\mathrm{B}_{\mathrm{N}^{-}} \mathrm{Y}\right) / 1.772+0.5$
$\mathrm{C}_{\mathrm{r}}=\left(\mathrm{R}_{\mathrm{N}^{-}} \mathrm{Y}\right) / 1.402+0.5$

\subsubsection{The CIE XYZ system}

It is at the root of all colorimetry. It is defined such that all visible colors can be defined using only positive values, and, the $\mathrm{Y}$ value is luminance.

$\mathrm{R}=3.24 X-1.537 \mathrm{Y}-0.498535 \mathrm{Z}$
$\mathrm{G}=-0.969 X+1.875 \mathrm{Y}+0.0415 \mathrm{Z}$
$\mathrm{B}=0.0556 \mathrm{X}+-0.204 \mathrm{Y}+1.057 \mathrm{Z}$

4.1.7 CIELUV is based on an attempt to define an encoding which is perceptually more uniform. To convert RGB to LUV First convert RGB to XYZ and then from $X Y Z$ to LUV as given below.

$u^{\prime}=\frac{4 X}{X+15 Y+3 Z} \quad$ and $v^{\prime}=\frac{9 Y}{X+15 Y+3 Z}$

$L= \begin{cases}16{\frac{Y}{Y_{n}}}^{\frac{1}{3}}-16, & \frac{Y}{Y_{n}}>\left(\frac{6}{29}\right)^{3} \\ \left(\frac{29}{3}\right)^{3}\left(\frac{Y}{Y_{n}}\right) & \frac{Y}{Y_{n}} \leq\left(\frac{6}{29}\right)^{3}\end{cases}$

Then

$$
\begin{aligned}
& \mathrm{U}=13 \mathrm{~L}\left(\mathrm{u}^{\prime}-\mathrm{u}^{\prime} \mathrm{n}\right) \\
& \mathrm{V}=13 \mathrm{~L}\left(\mathrm{v}^{\prime}-\mathrm{v}^{\prime} \mathrm{n}\right)
\end{aligned}
$$

\subsubsection{CIELAB}

$\mathrm{LAB}$ color is designed to approximate human vision. It aspires to perceptual uniformity, and its L component closely matches human perception of lightness. It can thus be used to make accurate color balance corrections by modifying output curves in $\mathrm{A}$ and $\mathrm{B}$ components, or to adjust the lightness contrast using the $\mathrm{L}$ component.

The forward transformation

$$
\begin{aligned}
& L=116 f\left(\frac{Y}{Y_{n}}\right)-16 \\
& A=500\left[f\left(\frac{X}{X_{n}}\right)-f\left(\frac{Y}{Y_{n}}\right)\right] \\
& \mathrm{B}=200\left[f\left(\frac{Y}{Y_{n}}\right)-f\left(\frac{Z}{Z_{n}}\right)\right]
\end{aligned}
$$




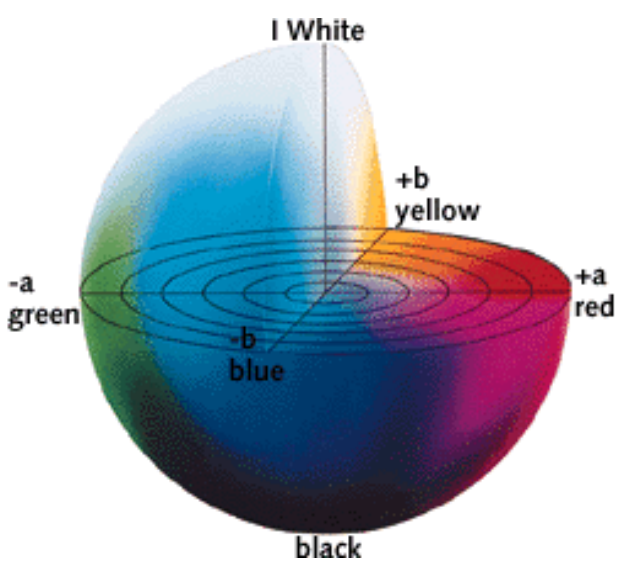

Fig.6: $L * a * b *$ color space

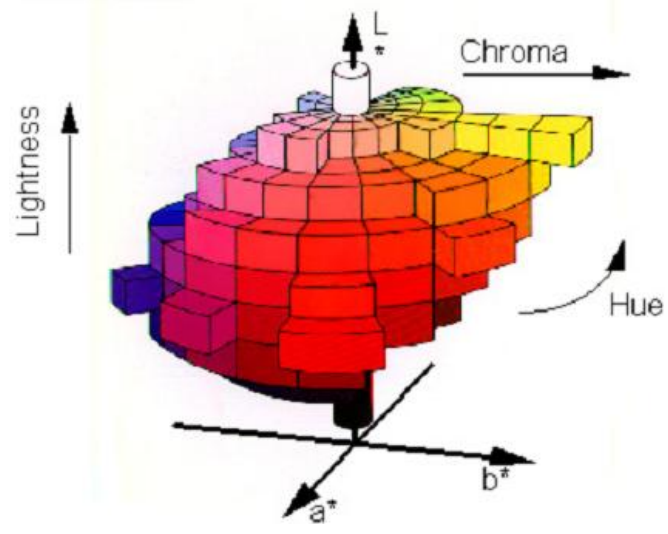

Fig.7: TheCIELab colour space

\subsubsection{CIELCH}

$\mathrm{LCH}$ is LAB in polar coordinate. To convert RGB to $\mathrm{LCH}$ first convert RGB to $\mathrm{LAB}$ and then to $\mathrm{LCH}$. Given LAB components:
$\mathrm{L}=\mathrm{L}$
$\mathrm{C}=\left(\mathrm{A}^{2}+\mathrm{B}^{2}\right)^{\wedge 1 / 2}$
$\mathrm{H}=\tan ^{-1}(\mathrm{~A} / \mathrm{B})$
$\mathrm{H}$ is in degrees, not radians. If $\mathrm{H}<0^{\circ}$, add $360^{\circ}$ to
it. If $\mathrm{H} \geq 360^{\circ}$, subtract $360^{\circ}$ from it.[5]

\section{APPLICATION SPECIFIC COLOR SPACES}

\subsection{Quantization}

There are several color spaces, existing for a variety of reasons, such as Munsell HVC, RGB, decorrelated RGB (DRGB), CIEXYZ, CIELAB, CIELUV, CIELHC, and Helmoltz chromaticity coordinates.

To achieve satisfying quantization quality, choosing a perceptually uniform color spaces is necessary. Perceptual uniformity means that two color pairs which are equal in distance in a color space are perceived as equal in distance by viewers. Compared with other conventional color spaces, the Munsell

color space has better perceptual uniformity.

\subsection{Segmentation}

RGB space and the spaces derived from RGB by linear transformations are generally not suitable for color scene segmentation and analysis because of the high correlation among their components. Hence it is impossible to evaluate the similarity of two colors from their distance in those spaces, while the spaces derived from RGB by nonlinear transformations suffer from singularity, which may create discontinuities in the representation of colors and some components are numerically unstable.

The method to build a hybrid color space is proposed by Nicolas Vandenbroucke, in which the correlation between the components is low, and which is adapted to the best discrimination of pixel classes[6]

\subsection{Skin detection, face recognition}

Some of researchers used a color space in a specific application in the computer vision as skin detection in HSV, face recognition in new space based on K-L1 transform , lip tracking into the CIELAB, CIELUV color spaces, object recognition into illumination invariant type of RGB color space, Image restoration into the CIELAB color space, and so on[3]

\subsection{Face recognition}

The double-zero-sum(DZS) color spaces are more powerful than other color spaces for Face representation and recognition. [7]

\subsection{Pharmaceutical packaging}

In pharmaceutical packaging, tablet inspection is an important part of safety and quality standard. Image segmentation is the key process for visual inspection. To improve accuracy and robustness of segmentation a method to construct a hybrid color space for the color tablet images by selecting a set of color components can be used. Such space leads to a better discriminating power than classical color spaces,

Experimental results demonstrate that edges of tablets are accurately extracted in segmented image based on hybrid color space. Instead of searching a best color space for segmentation, the method to construct hybrid color space, by selecting a set of color components of classical spaces that leads to best discrimination power, can be used for automatic visual inspection system of color tablet.

Experiments demonstrate that in both gray level and RGB spaces, there is no effective method to overcome the influence of varying refraction and reflect in both color [6]tablets and soleplate area, let alone area with highlighted reflection, which results in the edge between tablet and soleplate is not precise and speckle in the segmented image.

It is difficult for classifier to detect defect. On the other hand, in hybrid color space, tablet edges are accurate, and problems like non-uniform illumination and highlight can be solved effectively.

\subsection{Change detection}

Most change detection methods are based on gray-level images. A gray-level image is regarded as a 1-D projection of three channels of color images. Therefore, more precise change detection results are expected by utilizing color information. Common applications for change detection include tracking moving objects, video surveillance systems, traffic monitoring and silhouette detection. In this case, CIELAB and CIELCH show better results than other color spaces[8]

\section{CONCLUSION}

In this paper comprehensive investigation is made for the different colour spaces used in image processing. The choice of the colour space can be a very important decision which can dramatically influence the results of the processing. The 
knowledge of various colour spaces can ease the choice of the appropriate colour space. This paper gives broader view for dealing with different colour spaces as per the applications

Many Image processing applications convert colour image into grayscale and uses it for further processing, but for certain application useful information is available in the form of colour. Different colour models may be used to create the feature vectors for the Images and also the relationships and correlations between different colour components of same color space may be effective for classification.

\section{REFERENCES}

[1] Xie Yong1,2, Wang Yaonan1, Peng Tao2 "Influences Of Color Spaces On Printing Defect Classification" , 978-1-4244-2723-9/09/\$25.00 C 2009ieee

[2] Yuanyuan Pu, Xiaomin Wei, Yi Su, Zhengpeng Zhao, Wenhua Qian, Dan Xu "Implementation And Research Of Multi-Method Color Transfer Algorithms In Different Color Spaces" ,2010 International Conference On Machine Vision And Human-Machine Interface

[3] Seyyed Meysam Hosseini , Hasan Farsi , Saber Amjadi "A Novel Color Space Creating Method Applied To Skin Color Detection" 987-0-7695-3565-4/09, 2009 Ieee.

[4] Imtnan-Ul-Haque Qazi, Olivier Alata, Jean-Christophe Burie "Choice Of A Pertinent Color Space For Color
Texture Characterization Using Parametric Spectral Analysis", 0031-3203/-See Front Matter \& 2010elsevierltd, Doi:10.1016/J.Patcog.2010.07.007

[5] Melkamu H. Asmare , 2vijanth S. Asirvadam , 3lila Iznita "Color Space Selection For Color Image Enhancement Applications" 978-0-7695-3594-4/09 $\$ 25.00$ (C) 2009 Ieee Doi 10.1109/Icsap.2009.39

[6] Cai Yejing Long Yonghong," "A New Method Of Hybrid Color Space And Its Application", 978-0-76953583-8/09 \$25.00 (C) 2009 Ieee, Doi 10.1109/Icmtma.2009.323

[7] Jianyang, Chengjunliu ,Jing-Yuyang A "What Kind Of Color Spaces Is Suitable For Color Face Recognition?", 0925-2312/See Front Matter \& 2010elsevierb.V. Doi:10.1016/J.Neucom.2010.02.005

[8] Youngbae Hwang, Jun-Sik Kim, Inso Kweon "Determination Of Color Space For Accurate Change Detection" 1-4244-0481-9/06/\$20.00 C2006 Ieee.

[9] George Paschos, "Perceptually Uniform Color Spaces For Color Texture Analysis: An Empirical Evaluation" Ieee Transactions On Image Processing, Vol.10, No.6, June2001 